\title{
Modeling bone marrow progenitor cell differentiation and susceptibility to HIV-I infection
}

\begin{abstract}
Human immunodeficiency virus type 1 (HIV-1) infection of the monocytic lineage is involved in the pathologic events associated with AIDS and HIV-1-associated dementia (HAD). Hematopoietic progenitor cells (HPCs) within the bone marrow are refractile to HIV-1 infection, while their progeny of the monocyte-macrophage lineage are susceptible. Previous studies, using phorbol-myristate-acetate (PMA) as a differentiating agent, have suggested that the $\mathrm{CD} 34^{+} / \mathrm{CD} 38+\mathrm{TF}-1$ cell line may be used as one model to study the differentiation processes of HPCs. In the present study, medium that has been conditioned by PMA-treated TF-1 cells but is devoid of any traces of PMA, was utilized to induce differentiation of TF-1 cells. The conditioned medium (CM) from this bone marrow-derived cell population is enriched with respect to numerous cytokines and induces differentiation and activation of TF-1 cells, as indicated by changes in the expression of CD34, CD38, and CD69 cell surface molecules. Furthermore, treatment with CM was also shown to induce the expression of CCR5 and CXCR4, while maintaining the expression of CD4, which was ultimately correlated with increased susceptibility to HIV-1. Additionally, the activation of the TF-1 cells was shown to lead to increased LTR activity, with specificity protein ( $\mathrm{Sp}$ ) and nuclear factor kappa-light-chain-enhancer of activated B cells) NF-kB factors playing a crucial role in HIV-1 long terminal repeat (LTR)-mediated transcription and possibly overall TF-1 permissivity. Interleukin (IL)-1 $1 \beta$, which is elevated in the CM, recapitulates some of the CM effects. In summary, these studies suggest that the TF-1 cell line could serve as a model to study the susceptibility of bone marrow progenitor cells to HIV-1 infection.
\end{abstract}

Keywords: TF-1, monocyte, conditioned medium, IL-1b, CD34, CD38
Volume I Issue 2 - 2014

\author{
Brian Wigdahl,' Aikaterini Alexaki, ${ }^{2}$ \\ Shane J Quiterio J Quiterio, ${ }^{3}$ Michael R \\ Nonnemacher Nonnemacher, ${ }^{3}$ Sonia Shah \\ Shah, ${ }^{3}$ Yujie Liu Liu, ${ }^{3}$ Anupam Banerjee \\ Banerjee, ${ }^{3}$ Luna Li Li, ${ }^{3}$ Vanessa Pirrone \\ Pirrone, ${ }^{3}$ Evelyn Kilareski Kilareski, ${ }^{3}$ \\ Constantinos Petrovas Petrovas, ${ }^{3}$ hendra \\ Passic Passic ${ }^{3}$ \\ 'Department of Microbiology and Immunology, and Center for \\ Molecular Virology and Translational Neuroscience, Institute for \\ Molecular Medicine and Infectious Disease, Drexel University \\ College of Medicine Philadelphia, USA \\ 'Department of Microbiology and Immunology, The Pennsylvania \\ State University, USA \\ ${ }^{3}$ Vaccine Research Center, National Institute of Allergy and \\ Infectious Diseases/NIH, USA
}

\begin{abstract}
Correspondence: Brian Wigdahl, Department of Microbiology and Immunology, Institute for Molecular Medicine and Infectious Disease, Drexel University College of Medicine, 245 N. I5th Street, MS \#I0I3A, Philadelphia, PA 19102, USA, Tel 2I5-7627598, Fax 215-762-1955, Email brian.wigdahl@drexelmed.edu
\end{abstract}

Received: May 06, 2014 | Published: May 28, 2014
Abbreviations: HIV-1, human immunodeficiency virus type 1; HAD, HIV-1- associated dementia; HPCs, hematopoietic progenitor cells; PMA, phorbol-myristate-acetate; CM, conditioned medium; SP, specificity protein; LTR, long terminal repeat; IL, interleukin; HAART, highly active antiretroviral Therapy; PBS, phosphate-buffered saline; GM-CSF, granulocyte macrophage-colony stimulating factor; FBS, fetal bovine serum; HBSS, hanks balanced saline solution

\section{Introduction}

Cells of the monocyte-macrophage lineage are a major target of human immunodeficiency virus type 1 (HIV-1), and play a crucial role in the dissemination of the virus and the development of disease symptomatology, AIDS, HIV-1-associated dementia (HAD), and perhaps other HIV-1-associated CNS disorders observed in patients treated with highly active antiretroviral therapy (HAART) for prolonged periods of time. ${ }^{1,2}$ Monocytes originate from the bone marrow, where pluripotent hematopoietic stem cells continuously divide and give rise to all cell lineages present in the peripheral blood compartment. The bone marrow has been shown to be dysfunctional in the pathology of AIDS, with thrombocytopenia, anemia, monocytopenia, and neutrocytopenia commonly observed. ${ }^{3,4}$

HIV-1 infection of bone marrow stromal cells, changes in the cytokine milieu of the bone marrow, and cytotoxic effects of HIV1 proteins are mechanisms involved in the impairment of HPC differentiation and growth, ultimately leading to hematopoietic defects. $^{5-7}$ Numerous co-infections as well as some lymphomas commonly observed in AIDS patients ${ }^{8}$ have been shown to further impact hematopoiesis in HIV-1-infected individuals. Direct HIV-1 infection of hematopoietic progenitor cells (HPCs) may contribute to hematopoietic abnormalities, however, the extent of infection in the bone marrow compartment remains controversial. ${ }^{9}$ Numerous studies have demonstrated the susceptibility of $\mathrm{CD} 34^{+}$bone marrow-derived cell populations to HIV-1 both in vivo and in vitro..$^{10-13}$ However, these studies have not necessarily considered that $\mathrm{CD} 34^{+}$bone marrow cells consist of a heterogeneous population, which includes hematopoietic stem cells, ancestral hematopoietic progenitor cells, as well as more mature hematopoietic progenitors and bone marrow progenitor stromal cells. Permissivity of $\mathrm{CD} 34^{+}$HPCs depends on the state of differentiation, with the committed progenitor cells being the most susceptible and the quiescent stem cells being the most refractile to HIV-1 infection. ${ }^{14,15}$ Interestingly, it has been shown that macrophage colony- stimulating factor (M-CSF) ${ }^{1}$ or the presence of infection with human herpesvirus-6 induces HIV-1 infection of $\mathrm{HPCs}^{16}$ through increased virus production or increased CD4 expression, respectively, emphasizing the crucial role that physiological changes in the bone marrow environment have on the HIV-1 susceptibility of this cellular compartment.

An intriguing possibility is that mature progenitor cells or cells that are committed to the monocyte lineage but still capable of a limited number of cell divisions, may be infected by HIV-1 while still in the bone marrow and subsequently migrate to the blood into peripheral tissues thereby contributing to the dissemination of virus. The study by Bailey et al. has provided evidence supporting this hypothesis, by showing that in some HIV-1-infected patients on HAART, there are one or two predominant plasma HIV-1 sequences that are rarely encountered in T cells. These sequences are found in the plasma over prolonged periods of time and it was hypothesized that a cell with 
proliferative capacity resistant to the cytopathic effects of HIV-1, such as a monocyte progenitor, could be responsible for the release of the virus in the blood. ${ }^{17}$ Trafficking of cells of the monocyte-macrophage lineage is of particular importance because of their ability to cross the blood brain barrier (BBB) and deliver the virus to the central nervous system (CNS) thereby contributing to the development of HIV-1-associated neurologic disease. ${ }^{18,19}$ There is a continuous renewal of the perivascular macrophages located on the parenchymal side of the CNS by bone marrow-derived monocytes. Trafficking to the brain is accelerated in cases of inflammation and potentially leads to acceleration of HAD during the later stages of HIV-1 infection as previously proposed. ${ }^{19}$

Herein, an in vitro model was developed to study differentiation of myeloid progenitor cells with respect to their ability to be infected by HIV-1. To do so, the TF-1 cell line was used; this cell line was derived from a patient with erythroleukemia and its proliferation has been shown to be dependent on the presence of either granulocyte macrophage-colony stimulating factor (GM-CSF) or interleukin (IL)$3 .^{20}$ These cells are $\mathrm{CD} 34^{+}$hematopoietic precursor cells blocked at an early stage of hematopoietic differentiation and express several erythroid and myeloid markers. ${ }^{21} \mathrm{TF}-1$ cells have been used by several investigators as a model of multipotent progenitor cell growth and differentiation, as they can respond by differentiation, proliferation, or apoptosis to a variety of cytokines and to selected chemical stimuli. Phorbol-myristate-acetate (PMA) stimulation leads to a profound differentiation of TF-1 cells into macrophage-like cells, ${ }^{20,21}$ and at the same time induces apoptosis within the non-adherent cells in the treated population. ${ }^{22,23}$ As a result, we sought to identify a procedure that would lead to a similar differentiated morphology and phenotype as PMA treatment but would not induce cell death and downregulation of key surface molecules involved in HIV-1 entry. ${ }^{24}$

The studies reported herein identify conditioned medium (CM) derived from PMA-treated cells as a treatment that can induce activation and differentiation of TF-1 cells towards a myeloid phenotype. CM, which does not contain any PMA, is physiologically more relevant than a chemical agent and does not induce apoptosis. Furthermore, treatment of TF-1 cells with CM leads to upregulation of CCR5 and CXCR4 but unlike PMA, it did not result in CD4 downregulation. The concurrent expression of the HIV-1 receptor and co-receptors could account for the increased susceptibility of these cells to HIV-1 infection. Additionally, CM-induced differentiation of TF-1 cells resulted in an enhanced activation of the HIV-1 long terminal repeat (LTR). Finally, cytokines were measured in the CM and showed IL-1 $\beta$ and others to be increased with IL-1 $\beta$, in part, being able to recapitulate the cellular phenotype observed with $\mathrm{CM}$ treatment. The studies presented herein, suggest that the TF-1 cell line may serve as a useful model to study the effect of differentiation on the susceptibility of bone marrow progenitor cells to HIV-1 infection. CM treatment is used as physiologically relevant method of stimulating TF-1 cells through autocrine- and/or paracrine-based signals to provide additional evidence that promonocytic progenitor cells could be a target for HIV-1 within the context of a bone marrow environment.

\section{Materials and methods}

\section{Cell culture and cell treatments}

The TF-1 CD34 ${ }^{+}$erythro-myeloid leukemia cell line (ATCC, Manassas, VA) was grown in RPMI 1640medium (ATCC) supplemented with $10 \%$ heat-inactivated fetal bovine serum (FBS;
HyClone, Logan, UT), penicillin $(100 \mathrm{U} / \mathrm{mL})$, streptomycin $(100 \mu \mathrm{g} /$ $\mathrm{mL}$ ), and recombinant human granulocyte-macrophage-colony stimulating factor (rhGM-CSF, 2ng/ml; eBioscience, San Diego, CA). The cells were maintained at a maximum density of $4 \times 10^{5}$ cells $/ \mathrm{ml}$, at $37^{\circ} \mathrm{C}$ in $5 \% \mathrm{CO} 2$ at $90 \%$ relative humidity. TF-1 cells were treated with PMA $(300 \mathrm{ng} / \mathrm{ml})$ for $15 \mathrm{~min}$ at a cell density of $2 \times 10^{6} \mathrm{cell} / \mathrm{s} / \mathrm{ml}$, at $37^{\circ} \mathrm{C}$. The cells were then washed twice with wash buffer phosphatebuffered saline (PBS) with $3 \%$ FBS, re suspended at a density of $5 \times$ $10^{5}$ cells $/ \mathrm{ml}$ and incubated at $37^{\circ} \mathrm{C}$. CM was made by treating TF-1 cells with PMA and washing 2, 4, and 6 hours after the 15-min PMA treatment with wash buffer. The cells were then incubated for 24 hours, and the medium was collected, centrifuged at $2000 \mathrm{rpm}$ for $5 \mathrm{~min}$, and filtered through a $0.2 \mu \mathrm{m}$ pore filter (Millipore, Billerica, MA). TF-1 cells were treated with CM diluted 1:2 with fresh medium. To make twice conditioned medium (2CM), medium from TF-1 cells that had been treated with $\mathrm{CM}$ for 24 hours was collected, centrifuged at $2000 \mathrm{rpm}$ for $5 \mathrm{~min}$, and filtered through a $0.2 \mu \mathrm{m}$ pore filter. TF-1 cells were treated with $2 \mathrm{CM}$ diluted 1:2 with fresh medium prior to experimentation. Recombinant human cytokines, IL-1 $\beta$, MIP- $1 \alpha$, MIP-1 $\beta$, RANTES, and IL-8 (R\&D systems, Minneapolis, MN) were used at a concentration of $30 \mathrm{ng} / \mathrm{ml}$.

\section{Flow cytometry (FCM) analysis}

TF-1 cells were washed with FCM buffer Hanks balanced saline solution (HBSS) (Mediatech, Herndon, VA), 3\% FBS and 0.02\% $\mathrm{NaN} 3$ and aliquots of $5 \times 10^{5}$ cells were reacted with titrated amounts of conjugated antibody or Annexin V, the cell suspension was mixed gently, incubated on ice for $30 \mathrm{~min}$, and washed once with FCM buffer $(4 \mathrm{ml})$. FCM antibodies were obtained from eBioscience unless otherwise indicated. The following fluorochrome-conjugated FCM antibody combinations were used to determine cell surface expression of their respective proteins: (1) FITC-anti-CCR5 (R\&D Systems), PE-anti-CXCR4 (R\&D Systems), and PE-Cy5-anti-CD4; (2) FITCanti-CD69, PE-anti-CD38, and APC-anti-CD34 (BD Biosciences, San Jose, CA); (3) PE-anti-CD11b, APC-anti-CD14; and (4) FITCanti-CD13, PE-anti-CD64. Additionally, combinations of (1) APCAnnex in V, FITC-anti-Ki67 (BD biosciences) and (2) APC-Annex in $\mathrm{V}$, propidium iodine (PI) were used. When Annex in $\mathrm{V}$ was used, $\mathrm{CaCl}_{2}(2.5 \mathrm{mM})$ was added to all steps. Intracellular staining with anti-Ki67 was performed following surface staining; cells were permeabilized with Cytofix/Cytoperm buffer (BD Biosciences) for 20 min on ice, washed twice with $1 \mathrm{ml}$ of Perm/Wash (BD Biosciences), incubated for 30min with anti-Ki67, and washed again. Concentration matched isotype controls were used for each of the antibodies examined. Paraformaldehyde $(1 \%, 200 \mathrm{ml})$ was added to the cells following the last wash unless PI was used. PI was added to unfixed cells immediately before analysis. FCM analysis was performed utilizing a Calibur Flow Cytometer (BD Biosciences) and the results were analyzed using Flowjo version 6.1.1 software (Tree Star, San Carlos, CA).

\section{HIV-I infection of TF-I cells}

Untreated cells and cells treated for 1,2, or 3days with CM were harvested with cellstriper (Mediatech) and seeded in 12-well plates with a cell density of $0.2 \times 10^{6}$ cells $/ \mathrm{ml}(1 \mathrm{ml} /$ well $)$. Untreated cells were seeded either in fresh medium or in 1:2 diluted CM. Cells were then infected with $4 \mu \mathrm{l} / \mathrm{ml}$ of HIV- 1 strains IIIB or BaL $\left(10^{5} \mathrm{TCID}_{50}\right.$ per $\mu \mathrm{L}$; Advanced Biotechnologies, Inc., Columbia, MD) per well. Two hours after infection, cells were washed with PBS, and reseeded in 12well plates. Untreated cells that were infected in the presence of CM were either seeded in fresh or 1:2 diluted CM. Twenty-four hours after 
the infection, the medium from each well was collected and cells were removed by centrifugation at $1100 \mathrm{rpm}$ for $5 \mathrm{~min}$. The supernatant was collected and HIV-1 p24 was quantified using an Alliance p24 ELISA kit as described by the manufacturer (PerkinElmer, Wellesley, MA).

\section{Cloning and site-directed mutagenesis}

Site-directed mutagenesis was performed on the HIV-1 IIIB/LAIpGL3 reporter vector, ${ }^{25}$ to incorporate knock out $(\mathrm{KO})$ mutations into the Sp sites I, II, and III, NF- $\kappa B$ sites I and II, and C/EBP sites I and II of the HIV-1 IIIB/LAI LTR. The following primers were used for sitedirected mutagenesis, the mutated nucleotide(s) is/are underlined: $\mathrm{Sp}$ site I KO (5'-CCT-GGG-CGG-GAC-TGG-AGA-GTG-GCG-AGCCCT-C-3'), Sp site II KO (5' TGG-AGG-CGT-GGC-CTG-AGCGGG-ACT-GGA-GAG-T-3'), Sp site III KO (5'GGG-ACT-TTCCAG-GGA-AGC-GTG-GCC-TGA-GCG-G-3'), NF-кB site I KO (5'-GAC-TTT-CCG-CTG-GGG-CCT-TTC-CAG-GGA-GGC-G-3'), NF- $\mathrm{kB}$ site II KO (5'-AGC-TTG-CTA-CAA-GGG-CCT-TTCCGC-TGG-GGC-C-3'), C/EBP site I KO (5'-ACT-GCT-GAC-ATCGAG-TTT-GCT-ACA-AGG-GAC-T-3'), and C/EBP site II KO (GAC-AGC-CGC-CTA-GCA-TTT-GAG-CAC-GTG-GCC-CGAGAG-3'). Sequences were confirmed by automated DNA sequencing (Seq8000/8800 Beckman Coulter, Fullerton, CA) and sequence analysis using Laser gene software (DNASTAR, Inc., Madison, WI) in the DNA Sequencing Laboratory of the Center for Molecular and Functional Genomics, Institute for Molecular Medicine and Infectious Disease, Drexel University College of Medicine.

\section{Transient expression analyses}

TF-1 cells $\left(2 \times 10^{6}\right)$ were transiently transfected with the Amaxa transfection system kit $\mathrm{V}$ as described by the manufacturer (Gaithersburg, MD). Cells were transfected with the firefly luciferase IIIB/LAI-pGL3-based construct (500 ng) in conjunction with the renilla luciferase pRL-TK internal control vector (100ng). Immediately after transfection, $10^{6}$ cells were either untreated or placed in $\mathrm{CM}$ and incubated for 16hours. Cells were washed with PBS, lysed with passive lysis buffer, and analyzed with luminometry (Dual-Glo luciferase assay system, Promega and Fluoroskan Ascent, Thermo Labsystems).

\section{Cytometric bead array}

The human Flex set for IL- $1 \beta$, TNF- $\alpha$, MIP- $1 \alpha$, MIP-1 $\beta$, G-CSF, IL-4, IL-6, IL-8, IL-10, MCP-1, and RANTES was used in a cytometric bead array as described by the manufacturer (BDbiosciences). In brief, undiluted untreated medium (UTM), CM, 2CM and Flex set standards were mixed with Flex set capture beads and incubated for $1 \mathrm{hr}$ at RT. $\mathrm{PE}$ detection reagent was added to the sample and incubated for $2 \mathrm{hr}$ at RT. Samples were washed with wash buffer (BDbiosciences) and analyzed with the BD LSR II Flow Cytometer. Concentrations of experimental samples were calculated based on comparison to the standard curves.

\section{Results}

\section{CM treatment induces differentiation and activation of TF-I cells}

Previous studies have demonstrated that continuous PMA treatment of TF-1 cells induced differentiation and activation of TF-1 cells, ${ }^{26}$ however, this was also accompanied by an increase in cell death. ${ }^{23}$. To identify an experimental approach to differentiate the TF-1 cell population under less cytotoxic conditions, a CM treatment was used to explore the possibility that factors secreted in the medium following PMA stimulation could be responsible for at least part of the observed alterations in TF-1 cell morphology. CM was prepared by treating TF-1 cells with a 15-min PMA pulse, washing the cells extensively to ensure that there was no PMA carry-over (see Materials and Methods), and incubating the cells for 24hours. High performance liquid chromatography (HPLC) analysis was performed on the $\mathrm{CM}$ to conclusively show that no trace of PMA was present (Mass Spectrometry Research Core, The Pennsylvania State University College of Medicine, Hershey, PA). The limit of detection of the assay is $0.5 \mathrm{ng} / \mathrm{ml}$ while the lowest PMA concentration which leads to a measurable effect is $5 \mathrm{ng} / \mathrm{ml}$ (data not shown). CM treatment, similar to the PMA pulse treatment, induced adherence of the cells; 24 and 72 hours following treatment, the adherent cells were collected and analyzed for surface molecule expression. PMA pulse treatment and $\mathrm{CM}$ treatment induced differentiation and activation to a similar extent (Figure 1). CD34, a progenitor cell marker, was down-regulated by both treatments at 72hours, suggesting a change in cellular metabolism consistent with the process of differentiation. CD38 was also down-regulated; this receptor has typically been shown to be absent from hematopoietic stem cells, but present in their multipotent progeny. As progenitor cells differentiate towards monocytes, CD38 has been shown to be down-regulated while in tissue macrophages it was typically absent. ${ }^{27} \mathrm{CD} 69$ expression was progressively increased with both treatments, indicating activation of the cells.

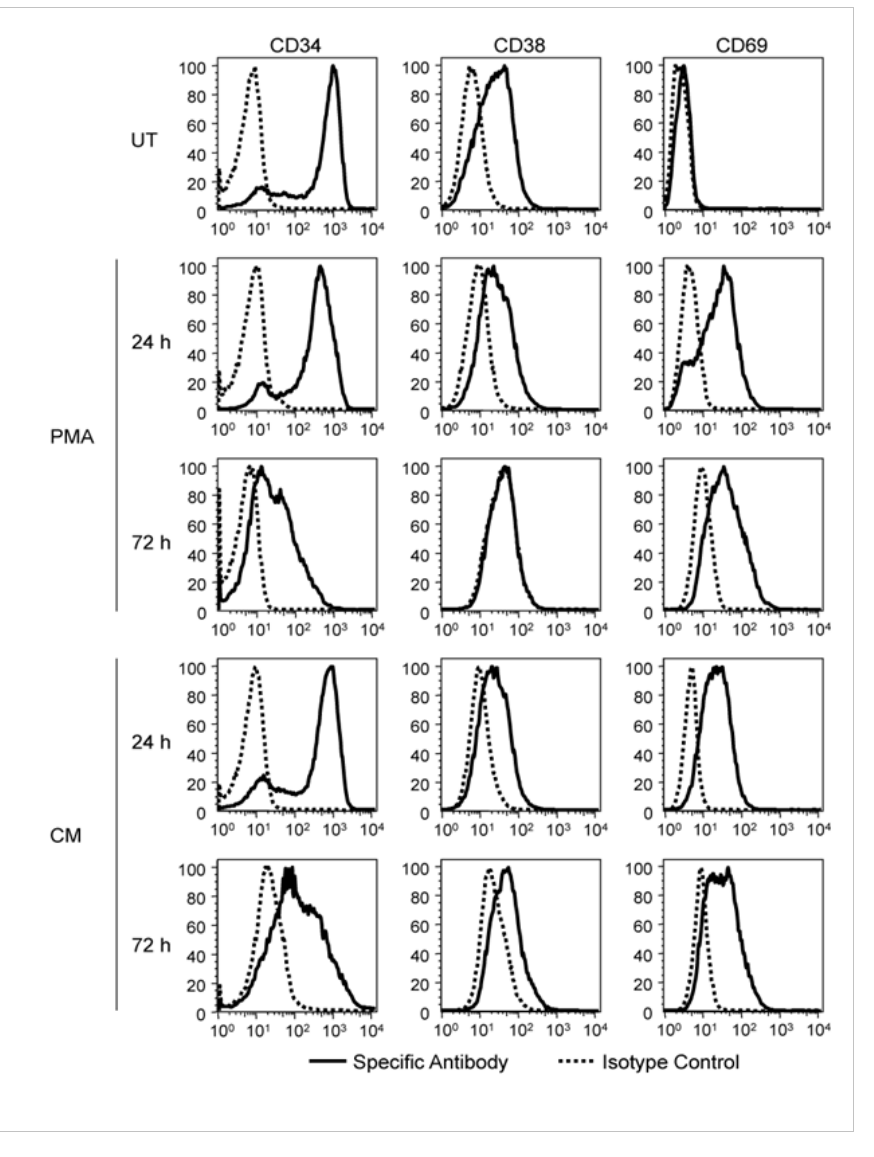

Figure I PMA- and CM-induced differentiation and activation of TF-I cells. TF-I cells were treated with a PMA pulse, with CM, or left untreated (UT). Twenty-four hours and 72 hours after treatment, untreated and adherent PMA and CM-treated cells were reacted with antibodies against CD34, CD38, and CD69. Surface expression was assessed by flow cytometry. A representative experiment out of three is shown. 


\section{CM treatment does not induce cell death}

PMA treatment of TF-1 cells has been reported to induce apoptotic death. ${ }^{23}$ As $\mathrm{CM}$ was able to induce differentiation to a similar extent as PMA, the level of cytotoxicity observed with the two treatments was compared. Cells were stimulated with a PMA pulse or with $\mathrm{CM}$, and cell growth was monitored over three days. Live cells were quantitated based on trypan blue dye exclusion. Untreated TF-1 cells grew exponentially during the 3 days they were observed (increasing by approximately 13-fold after 3days) (Figure 2A). Following PMA treatment, a decrease in the total number of TF-1 cells was observed at 24 hours with a further decrease at 72 hours (approximately 4-fold) At 24 hours, $57.7 \%$ of the PMA-treated cells were adherent, while at 72 hours $77.4 \%$ of live cells were adherent. CM treatment did not induce a decrease in cell number but rather resulted in a growth retardation, particularly at 72 hours. At 24 hours, $35.5 \%$ of the CMtreated cells were adherent, while at 72 hours $41.5 \%$ of the cells were adherent.
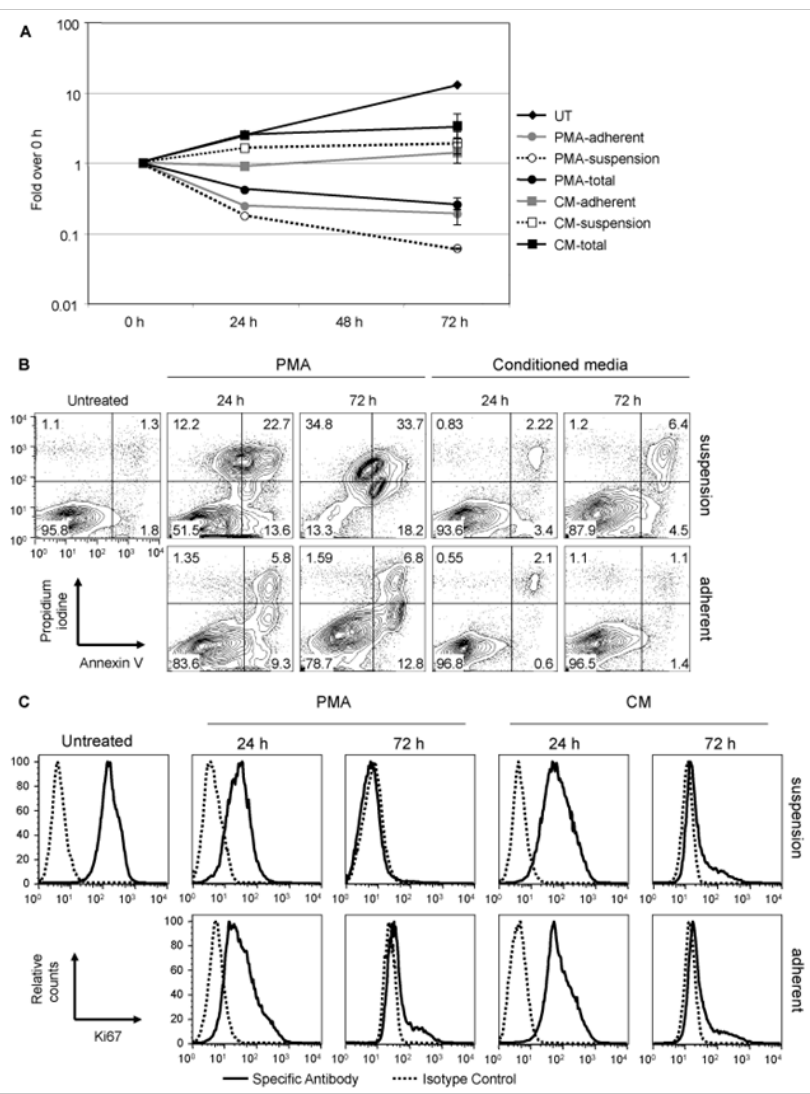

Figure 2 Cell death and proliferation arrest in TF-I cells caused by PMA and conditioned medium.TF-I cells were treated with a PMA pulse, with CM, or left untreated (UT). Twenty-four hours and 72 hours after treatment, cells were collected and adherent and suspension cells were counted $(A)$, and stained with Annexin $V$ and propidium iodine (B) or reacted with antibodies against $\mathrm{Ki} 67$ to detect intracellular protein (C). A representative experiment out of three is shown.

To determine whether the growth retardation observed after $\mathrm{CM}$ treatment was due to cell death or arrest in proliferation, untreated, PMA-treated, and CM-treated cells were reacted with fluorescently labeled anti-Ki67 antibody or annex in $\mathrm{V}$ and propidium iodine (PI) (Figure 2B \& 2C). Ki67 is a nuclear proliferation-associated molecule and is readily detected in cells that are actively proliferating. Annex in $\mathrm{V}$ is a marker of apoptosis, while PI is a necrosis marker. Single annex in $\mathrm{V}^{+}$or $\mathrm{PI}^{+}$cells are early apoptotic or early necrotic, respectively. Double-positive cells can be either late apoptotic or necrotic. Adherent and suspension PMA- and CM-treated cells were collected and stained separately. Untreated cultures had up to $4-5 \%$ annex in $\mathrm{V}^{+}$and/or $\mathrm{PI}^{+}$cells. PMA-treated cell cultures had considerably more cell death. At 24 hours, $48.5 \%$ of the cells in suspension were either apoptotic or necrotic. The early apoptotic cell population (PI-/annex in $\mathrm{V}^{+}$) was similar in size with the early necrotic cell population $\left(\mathrm{PI}^{+} /\right.$ annex in $\mathrm{V}-$ ). In the adherent fraction of PMA-treated cells at 24hours, there were very few early necrotic cells, while $16.1 \%$ of the cells were annex in $\mathrm{V}^{+}$. At 72 hours, the majority of cells in suspension (>85\%) were either necrotic or apoptotic, while about $20 \%$ of the adherent cells were annex in $\mathrm{V}^{+}$. In the $\mathrm{CM}$-treated cell population there were few annex in $\mathrm{V}^{+}$cells found in suspension, constituting about 5-6\% at 24 hours and $11 \%$ at 72 hours. In the adherent cell fraction, the amount of apoptosis and necrosis was comparable to the untreated population. Untreated TF-1 cells were positive for Ki67 indicating that they were proliferating. Both PMA and conditioned medium treatment induced down-regulation of Ki67 to moderate levels by 24hours, which resulted in a complete down-regulation of more than $90 \%$ of the cells by 72 hours of treatment. Collectively, these results indicate that the arrest in growth observed in CM-treated cells was primarily due to a decrease in proliferation and not to the limited extent of apoptotic death, while the reduction in the number of PMA-treated cells was due to both cell death and arrest in proliferation.
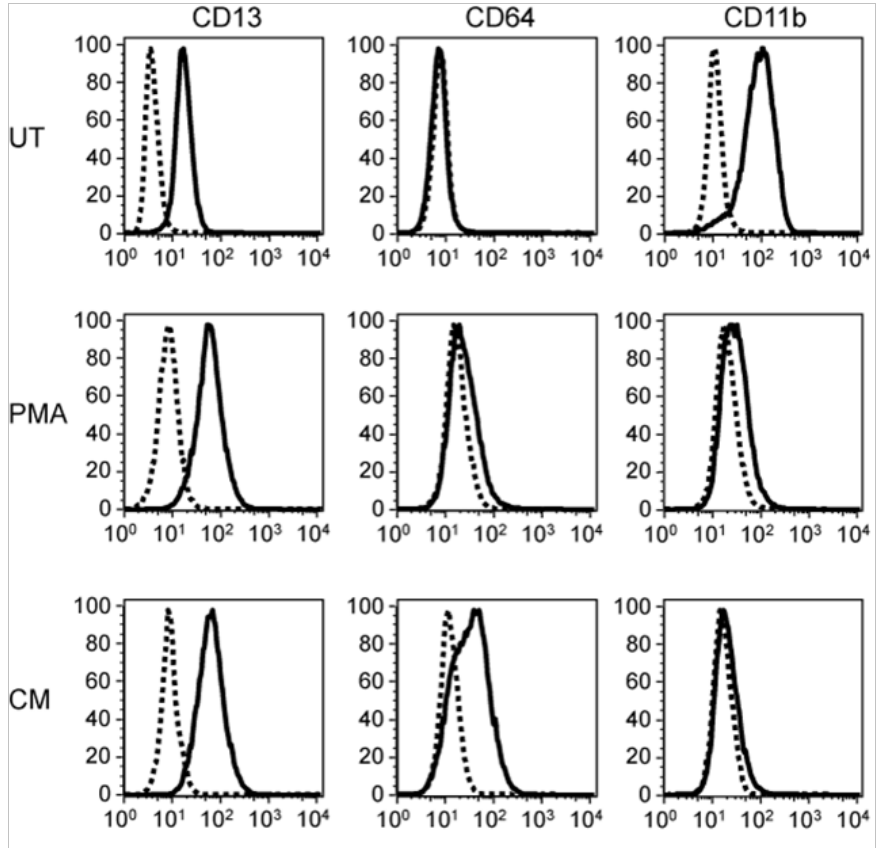

- Specific Antibody $\quad$...... Isotype Control

Figure 3 PMA and CM-induced changes in TF-I cell surface myeloid markers TF-I cells were treated with a PMA pulse or CM and stained for the indicated surface markers 72 hours later. A representative experiment out of three is shown.

\section{CM treatment induces the expression of myeloid markers on TF-I cells}

As previously demonstrated, the PMA pulse and CM treatment induced TF-1 cells to acquire a macrophage-like morphology. To confirm that CM treatment drives TF-1 cells towards the monocytemacrophage lineage, the myeloid surface markers CD13, CD64, and CD11b were examined 72hours after treatment (Figure 3). CD13 was detected on TF-1 cells and was demonstrated to be moderately 
increased after PMA and CM treatment. CD64, which is expressed on monocytes, macrophages, and can be induced following activation, was not detected on untreated TF-1 cells, but was shown to be modestly up-regulated on PMA-treated cells and strongly induced on CM-treated cells. CD11b, which was present on untreated cells, was down-regulated after both treatments.

A
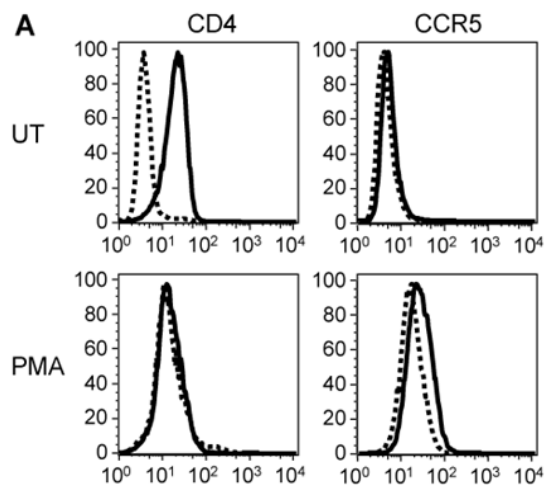

CM

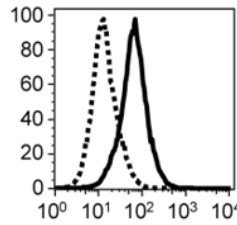

$2 \mathrm{CM}$

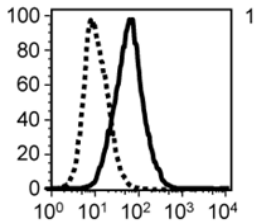

- Specific Antibody
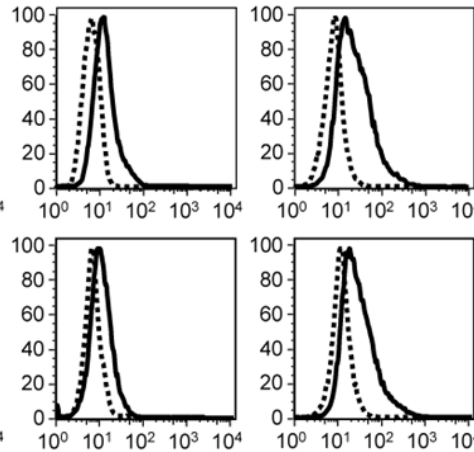

B

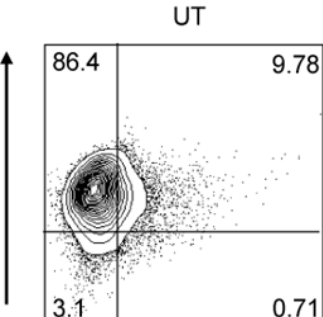

0.71

ป
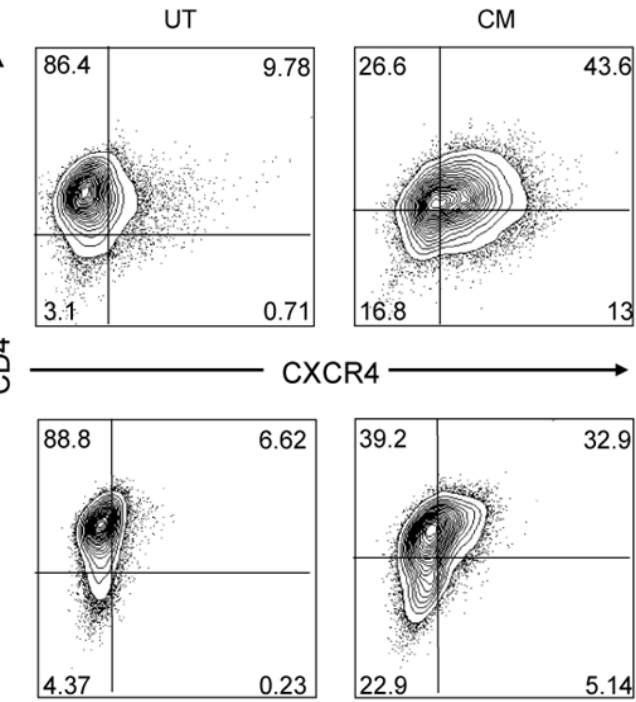

CXCR4

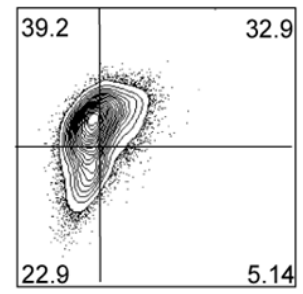

CCR5

Figure 4 PMA and CM-induced changes in HIV-I receptor and coreceptors on the TF-I cell surface.TF-I cells were treated with a PMA pulse, CM or 2CM and reacted with the specific monoclonal antibodies to detect the indicated surface markers 72 hours later. Histograms of the surface expression are shown (A). Contour plots exhibiting concurrent expression of CD4 and CXCR4 or CD4 and CCR5 in CM-treated TF-I cells are shown (B). A representative experiment out of three is shown.

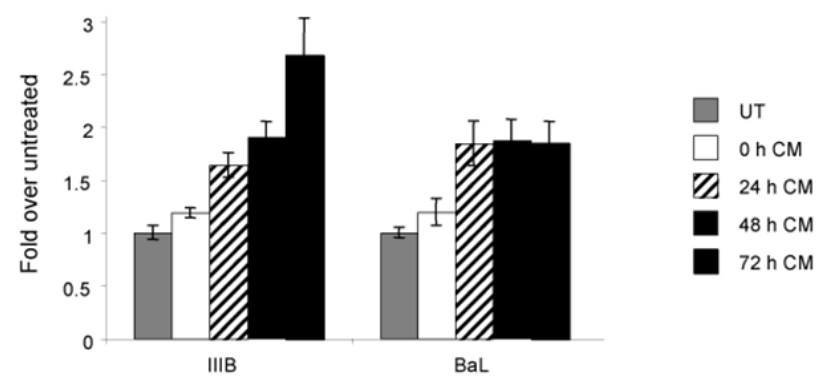

Figure 5 Increased HIV-I susceptibility of TF-I cells following CM-induced differentiation.TF-I cells were pre-treated with CM for $0,24,48$, or 72 hours and were then exposed to HIV-I in the presence of CM, or were never treated with conditioned medium (UT). Following 2 hours of exposure, cells were washed to remove the virus, and the medium was replaced. HIV-I p24 was quantitated in the supernatant 24 hours after virus exposure. In the UT samples infection with the IIIB strain of HIV-I lead on average to a p24 concentration of $102 \mathrm{ng} / \mathrm{ml}$, while infection with the BaL strain lead to a p24 concentration of $224 \mathrm{pg} / \mathrm{ml}$. P24 production is expressed as fold over the UT.
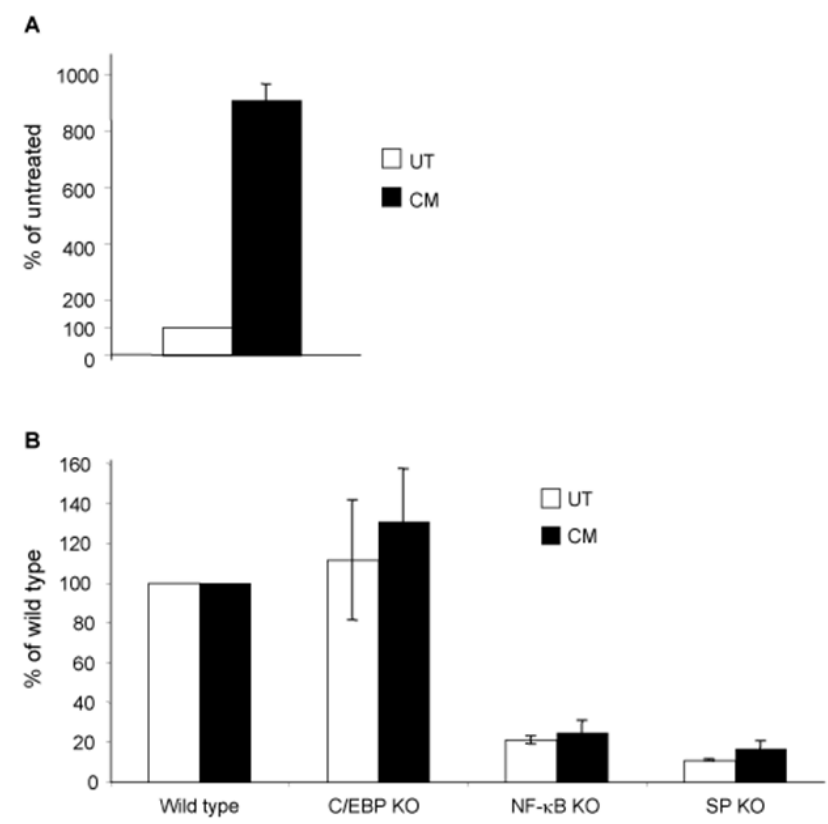

Figure 6 Increased HIV-I LTR activity in TF-I cells following CM treatment. TF-I cells were transiently transfected with the HIV-I IIIB/LAI-pGL3 construct (A) and the mutated C/EBP knockout (KO), NF-KB KO, or Sp KO HIV-I IIIB/ LAl-pGL3-based constructs (B). Following transfection, cells were incubated in CM or left untreated for 16 hours. Cells were collected and analyzed by luminometry. Results are shown as \% of the untreated control and represent the average of three independent experiments.

\section{CM treatment induces the HIV-I coreceptors CCR5 and CXCR4}

The expression of the HIV-1 receptor CD4 and co-receptors CXCR4 and CCR5 were also examined to determine whether differentiation of TF-1 cells could lead to changes in their expression that would increase the susceptibility of TF-1 cells to HIV-1 infection. Untreated TF-1 cells express the HIV-1 receptor on their surface but have very low to undetectable amounts of the co-receptors, and are therefore only moderately susceptible to HIV-1 infection. Seventytwo hours following the PMA pulse treatment, CXCR4 and to a lesser extent CCR5 expression was induced, however, CD4 expression was 
decreased to undetectable levels, which could potentially result in a decreased susceptibility to HIV-1 infection. The CM treatment did not induce a significant down-regulation of $\mathrm{CD} 4$ while it did result in a more pronounced CXCR4 and CCR5 up-regulation (Figure 4A). PMA treatment has been reported to result in the down-regulation of CD4 in various cell lines and primary cells, ${ }^{28,29}$ therefore, the absence of CD4 down-regulation in CM-treated cells was an indirect indication that PMA has been completely eliminated and was not present in the CM. To further exclude the possibility that traces of PMA in the CM may be partially responsible for the induction of the observed phenotype, twice conditioned medium ( $2 \mathrm{CM}$ ) was prepared and used to stimulate untreated TF-1 cells. $\mathrm{CM}$ and $2 \mathrm{CM}$ resulted in upregulation of the HIV-1 coreceptors to the same extent suggesting that factors secreted in the medium and not PMA are responsible for the induced phenotype. Double-staining of TF-1 cells with CD4 and CXCR4 or CCR5 antibodies clearly demonstrated that within the CM-treated cell population $43.6 \%$ of the cells were $\mathrm{CD}^{+} / \mathrm{CXCR}^{+}$and $32.9 \%$ of the cells were $\mathrm{CD}^{+} / \mathrm{CCR}^{+}$(Figure 4B), while within the untreated cell population the double-positive populations were $9.78 \%$ and $6.62 \%$, respectively. ${ }^{30}$

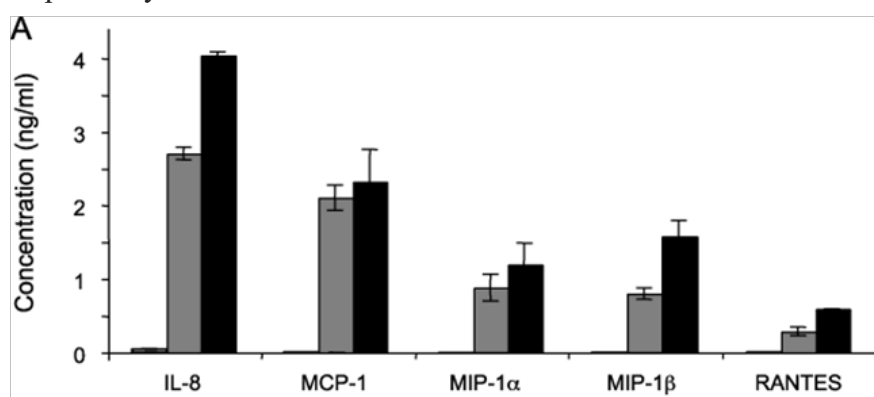

B

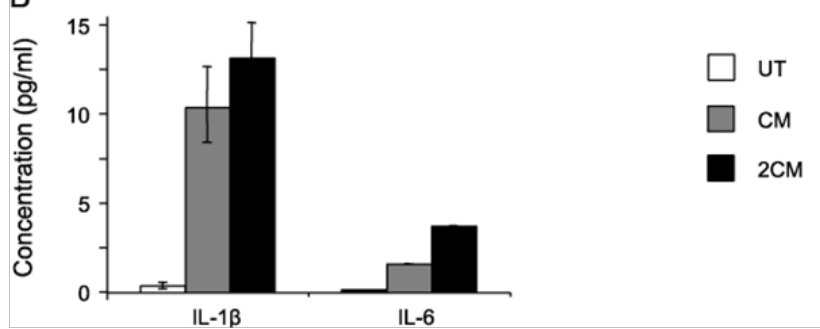

Figure $7 \mathrm{CM}$ contains several cytokines and chemokines. The presence of several cytokines was examined in the CM with a flow cytometry-based bead array. IL-8, MCP-I, MIP-I $\alpha$, MIP-I $\beta$, and RANTES concentration is shown in ng/ $\mathrm{ml}(\mathrm{A}), \mathrm{IL}-\mathrm{I} \beta$ and IL-6 concentration are shown in $\mathrm{pg} / \mathrm{ml}$ (B). TNF- $\alpha$, IL-4, and IL- I 0 were not detected in the CM (not shown).

\section{TF-I cells treated with CM are more susceptible to HIV-I infection}

To determine whether the simultaneous expression of HIV1 receptor and coreceptors, as observed after treatment with $\mathrm{CM}$, increased the susceptibility of TF-1 cells to infection, untreated TF-1 cells and cells treated for $0,24,48$, or 72 hours with $\mathrm{CM}$ were exposed to either the IIIB/LAI or BaL strains of HIV-1, which are X4- and R5-tropic, respectively. To determine whether CM has an effect on the events occurring during or following entry of the virus, cells that were not pretreated with $\mathrm{CM}$ (0 hour-pretreated), were incubated in $\mathrm{CM}$ following their exposure to the virus. After exposure to HIV-1 for 2hours, the virus was washed away, fresh medium or CM was replaced, and the cells were incubated for an additional 24hours. The presence of $\mathrm{CM}$, following exposure of the 0 hour-pretreated cells to the virus, had a very modest effect on the release of $\mathrm{p} 24$, as compared to untreated cells. In contrast, there was a significant increase in the susceptibility of CM-treated TF-1 cells to both HIV-1 strains examined when compared to the untreated TF-1 cells. Treatment of cells for 24,48 , or 72 hours prior to HIV-1 IIIB/LAI exposure resulted in p24 levels increasing 1.6, 1.9, or 2.7 fold, respectively (compared an average of UT $102 \mathrm{ng} / \mathrm{ml}$ p24 to 168,196 , and $275 \mathrm{ng} /$ $\mathrm{ml}$ ), demonstrating increasing susceptibility dependent on the time of pretreatment. Gradually increasing susceptibility, dependent on the time of pretreatment with CM, was not observed with the R5tropic HIV-1 BaL virus. Exposure of cells treated with CM to HIV-1 $\mathrm{BaL}$ for 24,48 , or 72 hours lead to approximately 1.85 fold more p24 (compared an average of UT $224 \mathrm{pg} / \mathrm{ml}$ to $390 \mathrm{pg} / \mathrm{ml}$ ) released into the supernatant when compared to untreated cells (Figure 5).

$\mathrm{CM}$ treatment up-regulates LTR-mediated transcription, with NF$\kappa \mathrm{B}$ and $\mathrm{Sp}$ transcription factors contributing to LTR activation. The bone marrow has been postulated to be a reservoir for latent HIV1 proviral DNA. To determine the effect of TF-1 differentiation on HIV-1 LTR activity, transient expression studies were conducted. Untreated TF-1 cells were transfected with the HIV-1 IIIB/LAI-pGL3 construct using the Amaxa transfection system, which has been shown to lead to a high level of transfection efficiency. Immediately after transfection, half of the cells were placed in regular medium and the other half in $\mathrm{CM}$ and incubated overnight. This procedure ensured that the same transfection efficiency was achieved in both untreated and CM-treated cells. CM treatment led to about 9-fold increase in luminescence compared to untreated samples (Figure 6A).

$\mathrm{C} / \mathrm{EBP}, \mathrm{NF}-\kappa \mathrm{B}$, and $\mathrm{Sp}$ binding site knockout configurations were incorporated into a HIV-1 IIIB/LAI-pGL3 construct to examine the contribution of these sites to basal and stimulated LTR activity (Figure 6B). Mutagenesis was performed on the two C/EBP sites, the two NF- $\kappa B$ sites, or the three $\mathrm{Sp}$ transcription factor binding sites. The parental HIV-1 IIIB/LAI LTR activity in untreated TF-1 cells was compared to the activities of the LTR with the mutated C/EBP, NF$\mathrm{B}$, or the Sp binding sites. There was no significant change in the LTR activity when the C/EBP sites were knocked out, while there was an $80 \%$ decrease in HIV-1 LTR activity when the NF-B sites were knocked out, and a 90\% decrease in LTR activity when the Sp sites were knocked out (Figure 6B). The parental HIV-1 IIIB/LAI-pGL3 and mutated IIIB/LAI-pGL3 were also transfected into TF-1 cells that were induced to differentiate with CM. In the differentiated TF-1 cells, there was again no significant change in LTR activity when the C/EBP sites were knocked out, while there was a $75 \%$ decrease in LTR activity when the NF- $\mathrm{kB}$ sites were knocked out, and an $83 \%$ decrease in LTR activity when the Sp sites were knocked out (Figure $6 \mathrm{~B})$. Collectively, the results have indicated that the C/EBP factors have little to no role in LTR transcription in TF-1 cells, while NF- $\kappa B$ and to an even greater extent $\mathrm{Sp}$ factors have a significant impact on LTR activity in both basal and stimulated conditions.

\section{A number of cytokines and chemokines are present in $\mathrm{CM}$}

Studies proceeded to examine the presence of cytokines in CM that could be involved in activating TF-1 cells during the CM treatment. A flow cytometry-based bead array was used for this purpose. The presence of IL-1 $\beta$, TNF- $\alpha$, MIP-1 $\alpha$, MIP-1 $\beta$, G-CSF, IL-4, IL-6, IL-8, IL-10, MCP-1, and RANTES was assessed in medium from UT cells, in CM, and 2CM. MIP- $1 \alpha$, MIP-1 $\beta$, IL-8, RANTES, and MCP- 1 were found in significant amounts both in $\mathrm{CM}$ and in $2 \mathrm{CM}$ while they were absent or at very low concentrations in UT. IL- $1 \beta$ and IL- 6 were also present in $\mathrm{CM}$ and $2 \mathrm{CM}$ but at very low concentrations. TNF- $\alpha$, IL-4, IL-10, and G-CSF were not detected in any of the media preparations examined (Figure 7). 


\section{IL-I $\beta$ treatment induces phenotypic changes on TF-I cells}

To further explore the effects of different cytokines present in CM, TF- 1 cells were treated with MIP-1 $\alpha$, MIP-1 $\beta$, IL-8, RANTES, MCP1 , or IL-1 $(30 \mathrm{ng} / \mathrm{ml})$ for up to 5 days and the expression of surface markers was determined by FCM. Treatment with MIP-1 $\alpha$, MIP-1 $\beta$, IL-8, RANTES, or MCP-1 did not induce any detectable changes in the expression of CD34, CD69, CXCR4, and CCR5 on TF-1 cells (data not shown). On the contrary, following a 5-day IL-1 $\beta$ treatment, CD34 and to a lesser extent CD38 expression was decreased, while a modest upregulation of CD69 in a subpopulation of cells was observed (Figure 8), collectively suggesting a change in cellular metabolism consistent with the process of differentiation. The expression of the HIV-1 receptor CD4 and co-receptors CXCR4 and CCR5 were also examined to determine whether IL-1 $\beta$-induced differentiation of TF-1 cells could lead to changes in their expression profile that would increase their susceptibility to HIV-1 infection. Following a 5-day IL-1 $\beta$ treatment, CXCR4 and to a lesser extent CCR5 expression were induced, CD4 expression remained unchanged, resulting in a concurrent expression of CD4 and at least one of the HIV-1 coreceptors in a significant proportion of the IL-1 $\beta$-treated cells (Figure 8).

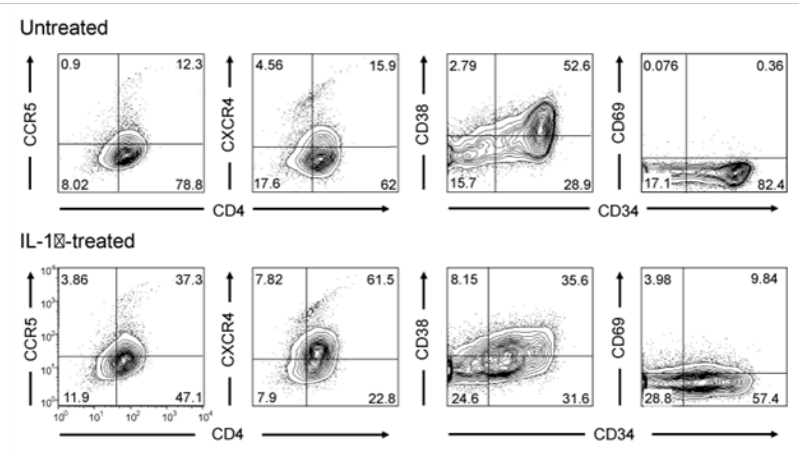

Figure $8 \mathrm{CM}$ contains several cytokines and chemokines. The presence of several cytokines was examined in the CM with a flow cytometry-based bead array. IL-8, MCP-I, MIP-I $\alpha$, MIP-I $\beta$, and RANTES concentration is shown in ng/ $\mathrm{ml}(\mathrm{A}), \mathrm{IL}-\mathrm{I} \beta$ and IL-6 concentration are shown in $\mathrm{pg} / \mathrm{ml}$ (B).TNF- $\alpha$, IL-4, and IL- I 0 were not detected in the CM (not shown).

\section{Discussion}

The ability of TF-1 cells to acquire a macrophage-like morphology has been described in numerous studies..$^{26,31,32}$ and recently their ability to produce IL- $1 \alpha$ and IL-1 $\beta$ was shown which suggested that PMA-stimulated TF-1 cells have also functional characteristics of macrophages ${ }^{33}$ However their surface molecule profile particularly, regarding myeloid and monocyte markers, had not been well characterized. Here we showed that untreated TF-1 cells are CD11b+, indicating that they are myeloid cells; the observation that at 72 hours following stimulation, CD11b expression was decreased, was somewhat puzzling. A possible explanation for this observation may center on what happens to neutrophils under similar circumstances. Potent stimulation of TF-1 cells may lead to transient up-regulation of CD11b, which is then followed by down-regulation of this cell surface marker. The appearance on the TF-1 cell surface of CD64 and the increase in CD13 surface expression, coupled with their macrophage-like morphology which were both observed with PMA and conditioned medium treatment. ${ }^{20,33}$ has strongly suggested that these cells are differentiating towards macrophages.

TF-1 cells, similar to bone marrow progenitor cells, can be infected by HIV-1 to a limited extent, possibly due to their low surface expression of the HIV-1 co-receptor CXCR4 and CCR5. ${ }^{9}$ Previous studies have shown that PMA-induced differentiation of TF-1 cells leads to morphologic and phenotypic changes and could be used as a model for myeloid progenitor cell differentiation. ${ }^{26}$ In the current study, the CM, which represents a spectrum of autocrine and paracrine signals, is proposed as an alternative to PMA treatment, being physiologically more relevant and inducing changes that are more favorable for the study of differentiation and susceptibility to HIV-1 infection. Unlike PMA, CM-induced differentiation does not promote cell death and does not induce CD4 down regulation; however, it leads to differentiation and up-regulation of CCR5 and CXCR4. As expected, the increased cell surface expression of HIV-1 co-receptors correlates with the increased susceptibility of TF-1 cells after differentiation as shown by increased p 24 production, and LTRdriven transcription is up-regulated in differentiated TF-1 cells as observed by transient transfection analysis. Susceptibility to the HIV$1 \mathrm{X} 4$-tropic virus IIIB gradually increased and was still increasing after 3days of CM treatment. On the contrary, susceptibility to the HIV-1 R5-tropic virus BaL was similarly increased in treated TF-1 cells, although it was not dependent on time of pre-treatment. The lack of response to the time of pre-treatment could be due to the secretion of factors in the medium such as MIP-1 $\alpha$, MIP-1 $\beta$, and RANTES that are the natural CCR5 ligands and have been shown to limit infection of the R5-tropic HIV-1 strains, ${ }^{34}$ including in $\mathrm{CD} 34^{+}$bone marrow progenitor cells. ${ }^{35}$ Similarly, TF-1 cells are shown to secrete MIP- $1 \alpha$, MIP-1 $\beta$, and RANTES following PMA stimulation, which could lead to a limited CCR5 availability and could explain why although the kinetics of CCR5 and CXCR4 appearance on the surface of the TF-1 cells are similar, the increase in susceptibility gradually reaches much higher levels with the $\mathrm{X} 4$-tropic virus.

MIP- $1 \alpha$, MIP-1 $\beta$, and RANTES, as well as IL- 8 and MCP-1 were found present in the $\mathrm{CM}$, however, these molecules individually did not appear able to reproduce the activating effects of CM. On the contrary IL-1 $\beta$, which is present in the CM in low concentration, is able induce some of the effects of CM. Nevertheless, the IL- $1 \beta$ concentration (30 $\mathrm{ng} / \mathrm{ml}$ ) that is needed to stimulate TF-1 differentiation and coreceptor expression is much higher that what is found in the CM (10 pg/ $\mathrm{ml}$ ), suggesting that this cytokine, although may be involved, is not exclusively responsible for the inducing effects of $\mathrm{CM}$. An alternative possibility is that several cytokines in the CM may act synergistically to induce the observed phenotype observed in the CM-treated cells. For example, it has been reported that IL-1 $\beta$ upregulates the expression of IL-3, IL-5, and GM-CSF receptors on TF-1 cells, leading to increased responsiveness to these cytokines. ${ }^{36}$ Similarly, IL- $1 \beta$ may increase responsiveness of TF-1 cells to other cytokines, or some cytokine in the CM may lead to upregulation of the IL- $1 \beta$ receptor expression on TF- 1 cells enabling a very small concentration of IL- $1 \beta$ to be more effective with respect to inducing physiological alterations in the TF-1 cell activation response. It is also possible that a yet unidentified factor may be present in the CM that may be responsible for the activation of TF-1 cells. In this case, the factor would likely be common in the down-stream pathways of both CM and IL- $1 \beta$, as this would explain the similarities in the induced effects.

HIV-1 infection of bone marrow progenitors has been of particular interest due to the trafficking of these cells, following their maturation, to peripheral tissues and particularly the CNS where they can deliver HIV-1 and initiate events that can lead to HAD. It has been hypothesized that monocytes that are generated in a pathologic bone marrow environment could be more prone to migrate to the central nervous system (CNS) thus contributing to the pathogenesis of HIV-1 dementia. ${ }^{37}$ The cytokine milieu of the inflamed bone marrow 
could lead to activation of the monocyte progenitors, and induction of surface receptors that could promote their homing to the perivascular spaces of the brain. Interestingly, there is evidence indicating that HIV1 sequences derived from the white matter of the brain more closely resemble sequences from the bone marrow than from other tissues, ${ }^{38}$ suggesting that HIV-1 infection of monocytes in the bone marrow may be involved in the trafficking of the virus to the brain. Furthermore, it has been shown that bone marrow diffusivity in HIV-1-infected patients correlates with dementia severity. ${ }^{39}$ further supporting the possibility that events in the $\mathrm{BM}$ are linked to migration of monocytes to the CNS. Recent results coming from a bacterial infection model suggest that chemokines secreted from sites of inflammation deliver signals in the bone marrow that mediate the emigration of monocytes from the BM to the blood, ${ }^{40}$ these sites of inflammation could be either distant or within the BM. This study, although conducted with a different model, supports the concept that infection in the BM may have a crucial role in the dissemination of the virus. Infection of bone marrow progenitor cells by HIV-1 and trafficking to the CNS is of need of further exploration. The TF-1 progenitor cell line provides a model that could facilitate studies in this area however studies expanding to primary cell models are needed.

\section{Acknowledgments}

These studies were funded in part by the Public Health Service, National Institutes of Health, through grants from the National Institute of Neurological Disorders and Stroke, NS32092 and NS46263, the National Institute of Drug Abuse, DA19807 (Brian Wigdahl, Principal Investigator), National Institute of Mental Health Comprehensive NeuroAIDS Core Center (CNAC),P30 MH-092177 (KamelKhalili, PI; Brian Wigdahl, PI of the Drexel subcontract), and under the Ruth L. Kirschstein National Research Service Award 5T32MH079785 (Jay Rappaport, PI; Brian Wigdahl, PI of the Drexel subcontract). The contents of the paper are solely the responsibility of the authors and do not necessarily represent the official views of the NIH.

\section{Conflicts of interest}

Author declares there are no conflicts of interest.

\section{Funding}

None.

\section{References}

1. Alexaki A, Liu Y, Wigdahl B. Cellular reservoirs of HIV-1 and their role in viral persistence. Curr HIV Res. 2008;6(5):388-400.

2. Gendelman HE, Meltzer MS. Mononuclear phagocytes and the human immunodeficiency virus. Curr Opin Immunol. 1999;(3):414-419.

3. Zhao X, Sun NC, Witt MD, et al. Changing pattern of AIDS:a bone marrow study. Am J Clin Pathol. 2004;121(3):393-401.

4. Isgro A, Aiuti A, Leti W, et al. Immunodysregulation of HIV disease at bone marrow level. Autoimmun Rev. 2005;4(8):486-490.

5. Lee CI, Cowan MJ, Kohn DB, et al. Simian immunodeficiency virus infection of hematopoietic stem cells and bone marrow stromal cells. $J$ Acquir Immune Defic Syndr. 2004;36(1):553-561.

6. Moses AV, Williams S, Heneveld ML, et al. Human immunodeficiency virus infection of bone marrow endothelium reduces induction of stromal hematopoietic growth factors. Blood. 1996;87(3):919-925.

7. Bahner I, Kearns K, Coutinho S, et al. Infection of human marrow stroma by human immunodeficiency virus-1 (HIV-1) is both required and sufficient for HIV-1-induced hematopoietic suppression in vitro:demonstration by gene modification of primary human stroma. Blood. 1997;90(5):1787-1798.
8. Moses A, Nelson J, Bagby GC. The influence of human immunodeficiency virus-1 on hematopoiesis. Blood. 1998;91(5):1479-1495.

9. Alexaki A, Wigdahl B. HIV-1 infection of bone marrow hematopoietic progenitor cells and their role in trafficking and viral dissemination. PLoS Pathog. 2008;4(12):e1000215.

10. Steinberg HN, Crumpacker CS, Chatis PA. In vitro suppression of normal human bone marrow progenitor cells by human immunodeficiency virus. J Virol. 1991;65(4):1765-1769.

11. Kitano K, Abboud CN, Ryan DH, et al. Macrophage-active colonystimulating factors enhance human immunodeficiency virus type 1 infection in bone marrow stem cells. Blood. 1991;77(8):1699-1705.

12. Folks TM, Justement J, Kinter A, et al. Characterization of a promonocyte clone chronically infected with HIV and inducible by 13-phorbol-12myristate acetate. J Immunol. 1988;140(4):1117-1122.

13. Ruiz ME, Cicala C, Arthos J, et al. Peripheral blood-derived $\mathrm{CD}^{+}{ }^{+}$progenitor cells: $\mathrm{CXC}$ chemokine receptor 4 and $\mathrm{CC}$ chemokine receptor 5 expression and infection by HIV. J Immunol. 1998;61(8):4169-4176.

14. Weichold FF, Zella D, Barabitskaja O, et al. Neither human immunodeficiency virus-1 (HIV-1) nor HIV-2 infects most-primitive human hematopoietic stem cells as assessed in long-term bone marrow cultures. Blood. 1998;91(3):907-915.

15. Shen H, Cheng T, Preffer FI, et al. Intrinsic human immunodeficiency virus type 1 resistance of hematopoietic stem cells despite co-receptor expression. J Virol. 1999;73(1):728-737.

16. Furlini G, Vignoli M, Ramazzotti E, et al. A concurrent human herpesvirus -6 infection renders two human hematopoietic progenitor (TF-1 and $\mathrm{KG}-1$ ) cell lines susceptible to human immunodeficiency virus type-1. Blood. 1996;87(11):4737-4745.

17. Bailey JR, Sedaghat AR, Kieffer T, et al. Residual human immunodeficiency virus type 1 viremia in some patients on antiretroviral therapy is dominated by a small number of invariant clones rarely found in circulating CD4+ T cells. J Virol. 2006;80(13):6441-6457.

18. Lassmann H, Schmied M, Vass K, et al. Bone marrow derived elements and resident microglia in brain inflammation. Glia. 1993;7(1):19-24.

19. Fischer-Smith T, Croul S, Sverstiuk AE, et al. CNS invasion by $\mathrm{CD} 14^{+} / \mathrm{CD} 16+$ peripheral blood-derived monocytes in HIV dementia:perivascular accumulation and reservoir of HIV infection. $J$ Neurovirol. 2001;7(6):528-541.

20. Kitamura T, Tange T, Terasawa T, et al. Establishment and characterization of a unique human cell line that proliferates dependently on GM-CSF, IL-3, or erythropoietin. J Cell Physiol. 1989;140(2):323-334.

21. Quiterio S, Grant C, Hogan TH, et al. C/EBP- and Tat-mediated activation of the HIV-1 LTR in $\mathrm{CD} 34^{+}$hematopoietic progenitor cells. Biomed Pharmacother. 2003;57(1):49-56.

22. Lai JM, Hsieh CL, Chang ZF. Caspase activation during phorbol ester-induced apoptosis requires ROCK-dependent myosin-mediated contraction. J Cell Sci. 2003;116(Pt 17):3491-3501.

23. Lai JM, Lu CY, Yang-Yen HF, et al. Lysophosphatidic acid promotes phorbol-ester-induced apoptosis in TF-1 cells by interfering with adhesion. Biochem J. 2001;359(Pt 1):227-233.

24. Baran J, Kowalczyk D, Ozog M, et al. Three-color flow cytometry detection of intracellular cytokines in peripheral blood mononuclear cells:comparative analysis of phorbol myristate acetate-ionomycin and phytohemagglutinin stimulation. Clin Diagn Lab Immunol. 2001;8(2):303-313.

25. McAllister JJ, Phillips D, Millhouse S, et al. Analysis of the HIV-1 LTR NF-kappaB-proximal Sp site III:evidence for cell type-specific gene regulation and viral replication. Virology. 2000;274(2):262-277. 
26. Alexaki A, Quiterio SJ, Liu Y, et al. PMA-induced differentiation of a bone marrow progenitor cell line activates HIV-1 LTR-driven transcription. DNA Cell Biol. 2007;26(6):387-394.

27. Deaglio S, Mehta K, Malavasi F. Human CD38:a (r)evolutionary story of enzymes and receptors. Leuk Res. 2001;25(1):1-12.

28. Pelchen-Matthews A, Parsons IJ, Marsh M. Phorbol ester-induced downregulation of CD4 is a multistep process involving dissociation from $\mathrm{p} 561 \mathrm{ck}$, increased association with clathrin-coated pits, and altered endosomal sorting. J Exp Med. 1993;178(4):1209-1222.

29. Clapham PR, Weiss RA, Dalgleish AG, et al. Human immunodeficiency virus infection of monocytic and T-lymphocytic cells:receptor modulation and differentiation induced by phorbol ester. Virology. $1987 ; 158(1): 44-51$.

30. Weinstein-Oppenheimer C, Steelman LS, Algate PA, et al. Effects of deregulated Raf activation on integrin, cytokine-receptor expression and the induction of apoptosis in hematopoietic cells. Leukemia. 2000;14(11):1921-1938.

31. Hoang T, Paradis E, Brady G, et al. Opposing effects of the basic helix-loop-helix transcription factor SCL on erythroid and monocytic differentiation. Blood. 1996;87(1):102-111.

32. Choi HJ, Smithgall TE. HIV-1 Nef promotes survival of TF-1 macrophages by inducing Bcl-XL expression in an extracellular signalregulated kinase-dependent manner. J Biol Chem. 2004;279(49):5168851696.

33. Alexaki A, Banerjee A, Kilareski E, et al. IL-1b production by differentiating TF-1 bone marrow progenitor cells. Nagoya, Japan:Proceedings of the 8th International Congress of Neuroimmunology; 2007. pp. 353-359.
34. Cocchi F, DeVico AL, Garzino-Demo A, et al. Identification of RANTES, MIP-1 alpha, and MIP-1 beta as the major HIV-suppressive factors produced by CD8+ T cells. Science. 1995;270(5243):1811-1815.

35. Majka M, Rozmyslowicz T, Ratajczak J, et al. The limited infectability by R5 HIV of CD34(+) cells from thymus, cord, and peripheral blood and bone marrow is explained by their ability to produce beta-chemokines. Exp Hematol. 2000;28(12):1334-1342.

36. Kitamura T, Takaku F, Miyajima A. IL-1 up-regulates the expression of cytokine receptors on a factor-dependent human hemopoietic cell line, TF-1. Int Immunol. 1991;3(6):571-577.

37. Gartner S (2000) HIV infection and dementia. Science. 2000;287(5453):602-604.

38. Liu Y, Tang XP, McArthur JC, et al. Analysis of human immunodeficiency virus type $1 \mathrm{gp} 160$ sequences from a patient with HIV dementia:evidence for monocyte trafficking into brain. J Neuroviro. 2000; 6(Suppl 1):S70 S81.

39. Ragin AB, Wu Y, Storey P, et al. Bone marrow diffusion measures correlate with dementia severity in HIV patients. AJNR Am J Neuroradiol. 2006;27(3):589-592.

40. Serbina NV, Pamer EG. Monocyte emigration from bone marrow during bacterial infection requires signals mediated by chemokine receptor CCR2. Nat Immunol. 2006;7(3):311-317. 